Recombination dynamics in CdTe/CdSe type-Il quantum dots

This article has been downloaded from IOPscience. Please scroll down to see the full text article.

2008 Nanotechnology 19115702

(http://iopscience.iop.org/0957-4484/19/11/115702)

The Table of Contents and more related content is available

Download details:

IP Address: 140.112.113.225

The article was downloaded on 26/06/2009 at 11:27

Please note that terms and conditions apply. 


\title{
Recombination dynamics in CdTe/CdSe type-II quantum dots
}

\author{
Chun Hsiung Wang ${ }^{1}$, Tzung Te Chen ${ }^{1}$, Yang Fang Chen ${ }^{1}$, \\ Mei Lin $\mathrm{Ho}^{2}$, Chih Wei Lai ${ }^{2}$ and Pi Tai Chou ${ }^{2}$ \\ ${ }^{1}$ Department of Physics, National Taiwan University, Taipei 106, Taiwan \\ ${ }^{2}$ Department of Chemistry, National Taiwan University, Taipei 106, Taiwan \\ E-mail: yfchen@phys.ntu.edu.tw
}

Received 19 October 2007, in final form 27 December 2007

Published 19 February 2008

Online at stacks.iop.org/Nano/19/115702

\begin{abstract}
Recombination dynamics in CdTe/CdSe core-shell type-II quantum dots (QDs) has been investigated by time-resolved photoluminescence (PL) spectroscopy. A very long PL decay time of several hundred nanoseconds has been found at low temperature, which can be rationalized by the spatially separated electrons and holes occurring in a type-II heterostructure. For the temperature dependence of the radiative lifetime, the linewidth and the peak energy of PL spectra show that the recombination of carriers is dominated by delocalized excitons at temperatures below $150 \mathrm{~K}$, while the mixture of delocalized excitons, electrons and holes overwhelms the process at higher temperature. The binding energy of delocalized excitons obtained from the temperature dependence of the non-radiative lifetime is consistent with the theoretical value. The energy dependence of lifetime measurements reveals a third power relationship between the radiative lifetime and the radius of QDs, the light of which can be shed by the quantum confinement effect. In addition, the radiative decay rate is found to be proportional to the square root of excitation power, arising from the change of wavefunction overlap of electrons and holes due to the band bending effect, which is an inherent character of a type-II band alignment.
\end{abstract}

(Some figures in this article are in colour only in the electronic version)

\section{Introduction}

Recently, type-II quantum dots (QDs) have attracted a lot of interest due to their unique properties fundamentally different from type-I QDs because of the spatial separation of confined electrons and holes, such as the large bending effect $[1,2]$, long radiative lifetime [3] and large optical anisotropy [4]. With these, type-II QDs provide a great deal of potential applications, including laser memory elements and many others $[5,6]$. In particular, the inherent property of the spatially separated photo-excited electrons and holes in a typeII heterostructure makes these material systems suitable for the fabrication of solar cells, in which photocarriers can contribute directly to the induced current before recombination [7]. In yet another research field, type-II QDs have been exploited successfully as near-infrared (NIR) light sources for biomedical imaging in living cells by taking advantage of their large photostability and deeper light penetration because the unique band alignment offers an additional dimension to fine tune the emitted photon energy [8].

$\mathrm{CdTe} / \mathrm{CdSe}$ core-shell QDs represent a typical and wellknown example for type-II band alignment due to its accessible fabrication process $[9,10]$. In previous studies, the basic optical properties of photoluminescence (PL) spectra in $\mathrm{CdTe} / \mathrm{CdSe}$ core-shell QDs, including size dependence of PL spectra, band bending effect and exciton binding energy, have been investigated [11]. In spite of its unique properties being attractive for both academic and industrial interests, the recombination dynamics of type-II QDs is still not well documented. In this paper, comprehensive time-resolved photoluminescence (TRPL) measurements and steady-state PL spectra have been performed in an aim to advance our understanding of the carrier recombination dynamics in CdTe/CdSe QDs, which may then serve as a model system for the study of other materials with similar band alignment. Several salient and interesting results have been obtained 
through our investigation. For example, the PL decay time ( $\sim 60-230 \mathrm{~ns}$ ) in type-II CdTe/CdSe QDs is found to be much longer than that in CdSe/ZnS type-I QDs ( 1-10 ns) [12]. Both radiative and non-radiative lifetimes show a complicated behavior arising from the dissociation of excitons, the results of which can be understood quite well with the assistance of steady-state PL spectra [11]. In the energy-dependent TRPL experiment, it is found that the relationship between the radiative lifetime and the size of CdTe/CdSe QDs can be well described by the quantum confinement effect. Finally, the square root dependence of the radiative decay rate on excitation power can be attributed to the band bending effect due to the spatially separated electrons and holes, an inherent characteristic of a type-II band alignment.

\section{Experiment}

The CdTe/CdSe type-II QDs were synthesized by the chemical colloidal method [9]. To obtain the CdTe/CdSe core/shell nanoparticles, the precipitated CdTe QDs (0.020 g) were dispersed in tri-n-octylphosphine oxide (TOPO, $2.20 \mathrm{~g}$ ) and hexadecylamine (HAD, $1.26 \mathrm{~g}$ ) before being heated to $190^{\circ} \mathrm{C}$. In addition, $\mathrm{CdCl}_{2}(0.092 \mathrm{~g})$ was dissolved in $2.5 \mathrm{ml}$ of tri-nbutylphosphine (TBP) upon gentle heating (ca. $\left.80^{\circ} \mathrm{C}\right)$. After being cooled to room temperature, the resulting $0.2 \mathrm{M}$ solution was mixed with $2.5 \mathrm{ml}$ of a $0.2 \mathrm{M}$ solution of $\mathrm{Se}$ in TBP. With a syringe pump, this mixture was injected within $1 \mathrm{~h}$ into the reaction flask containing the core nanocrystals at 190-200 ${ }^{\circ}$ C. After the addition was completed, the crystals were annealed at $190^{\circ} \mathrm{C}$ for an additional $11.5 \mathrm{~h}$. The prepared $\mathrm{CdTe} / \mathrm{CdSe}$ QDs were further purified by centrifugation and re-precipitation twice from methanol. Based upon our high resolution transmission electron microscope, the structure of the sample consists of CdTe (core) with a radius of $2.7 \mathrm{~nm}, \mathrm{CdSe}$ (shell) with a thickness of $1.0 \mathrm{~nm}$ and TOPO $[9,10]$.

For the PL measurement, an Ar-ion laser with $514.5 \mathrm{~nm}$ wavelength was used as the excitation source. The PL spectra were recorded by a Spectra Pro 300i monochromator and detected by an InGaAs detector. The sample exhibits a bright near-IR (NIR) emission at room temperature, indicating the excellent quality of our studied materials. For the TRPL measurements in the nano-microsecond region, a second harmonic of an Nd:YAG laser (532 nm, $8 \mathrm{ns,} \mathrm{continuum}$ surelite II) was used as an excitation source. Emission decay was then detected by a NIR-sensitive photomultiplier tube (Hamamatzu R5509-72) operated at $-80^{\circ} \mathrm{C}$, which was cooled by liquid nitrogen; the signal was sent through an oscilloscope (Model TDS 3012, Tektronix), which was then averaged over 512 shots for further analyses. Data were analyzed using the nonlinear least squares procedure in combination with an iterative convolution method. After the removal of the instrument response function, a temporal resolution of $\sim 5 \mathrm{~ns}$ could be achieved. To control the measurement temperature, the sample was placed inside a closed-cycle He refrigerator (Model $22^{\circledR}$ Refrigerator, CTI-CRYOGENICS).

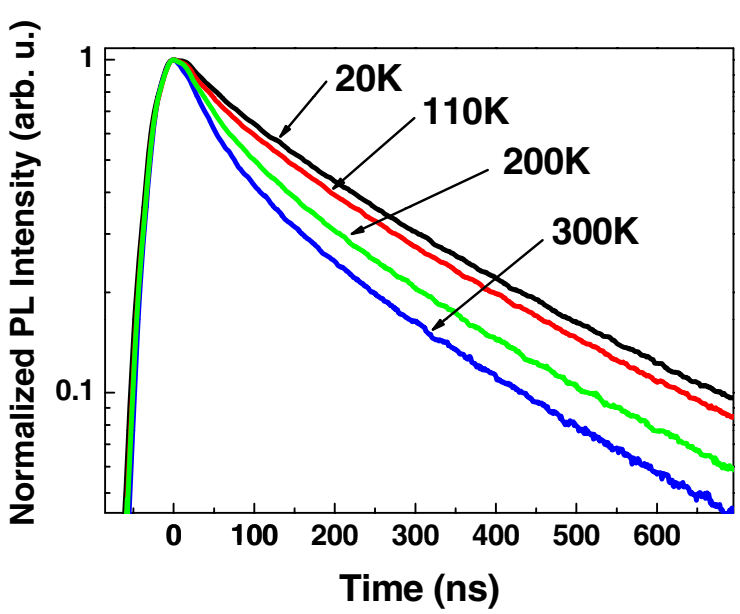

Figure 1. Time-resolved photoluminescence spectra of CdTe/CdSe core-shell type-II quantum dots at different temperatures.

\section{Results and discussions}

The PL decay curves of CdTe/CdSe QDs at different temperatures are shown in figure 1 , and the corresponding PL decay time can be obtained by fitting the stretched exponential equation [13]. It is found that the PL decay time decreases from 230 to $60 \mathrm{~ns}$ with increasing temperature from 20 to $300 \mathrm{~K}$. Obviously, the PL decay time (230 ns) measured at $20 \mathrm{~K}$ is much longer than that of type-I QDs with a typical value around several nanoseconds $[14,15]$. This slow recombination process can be easily understood based on the spatially separated electrons and holes in a type-II band alignment. With a more thorough examination, it is found that the measured lifetime consists of both of radiative and nonradiative components described by the expression [16]

$$
\frac{1}{\tau(T)}=\frac{1}{\tau_{\mathrm{rad}}(T)}+\frac{1}{\tau_{\mathrm{nrad}}(T)},
$$

where $\tau(T)$ is the measured PL decay time, $\tau_{\text {rad }}(T)$ is the radiative lifetime and $\tau_{\text {nrad }}(T)$ is the non-radiative lifetime. As for the steady-state approach, the quenching of the integrated PL intensity as a function of temperature can be described by equation [17]

$$
I(T)=I_{0} \frac{\tau(T)}{\tau_{\mathrm{rad}}(T)},
$$

where $I_{0}$ is the initial photo-excited carrier density. The quantum yield at $20 \mathrm{~K}$ can be described by the equation

$$
\eta(20 \mathrm{~K})=\frac{I(20 \mathrm{~K})}{I_{0}},
$$

where $\eta(20 \mathrm{~K})$ is the quantum yield at $20 \mathrm{~K}$ and $I(20 \mathrm{~K})$ is the integrated PL intensity at $20 \mathrm{~K}$. According to the quantum yield [10] and temperature dependence of the integrated PL intensity measurement, the obtained $\eta(20 \mathrm{~K})$ has a value of 0.4 , which is 13 times larger than the quantum yield at room temperature. Based on equations (1)-(3), the radiative lifetime $\tau_{\text {rad }}(T)$ and the non-radiative lifetime $\tau_{\text {nrad }}(T)$ can be deduced and the corresponding data are shown in figures 2(a) 

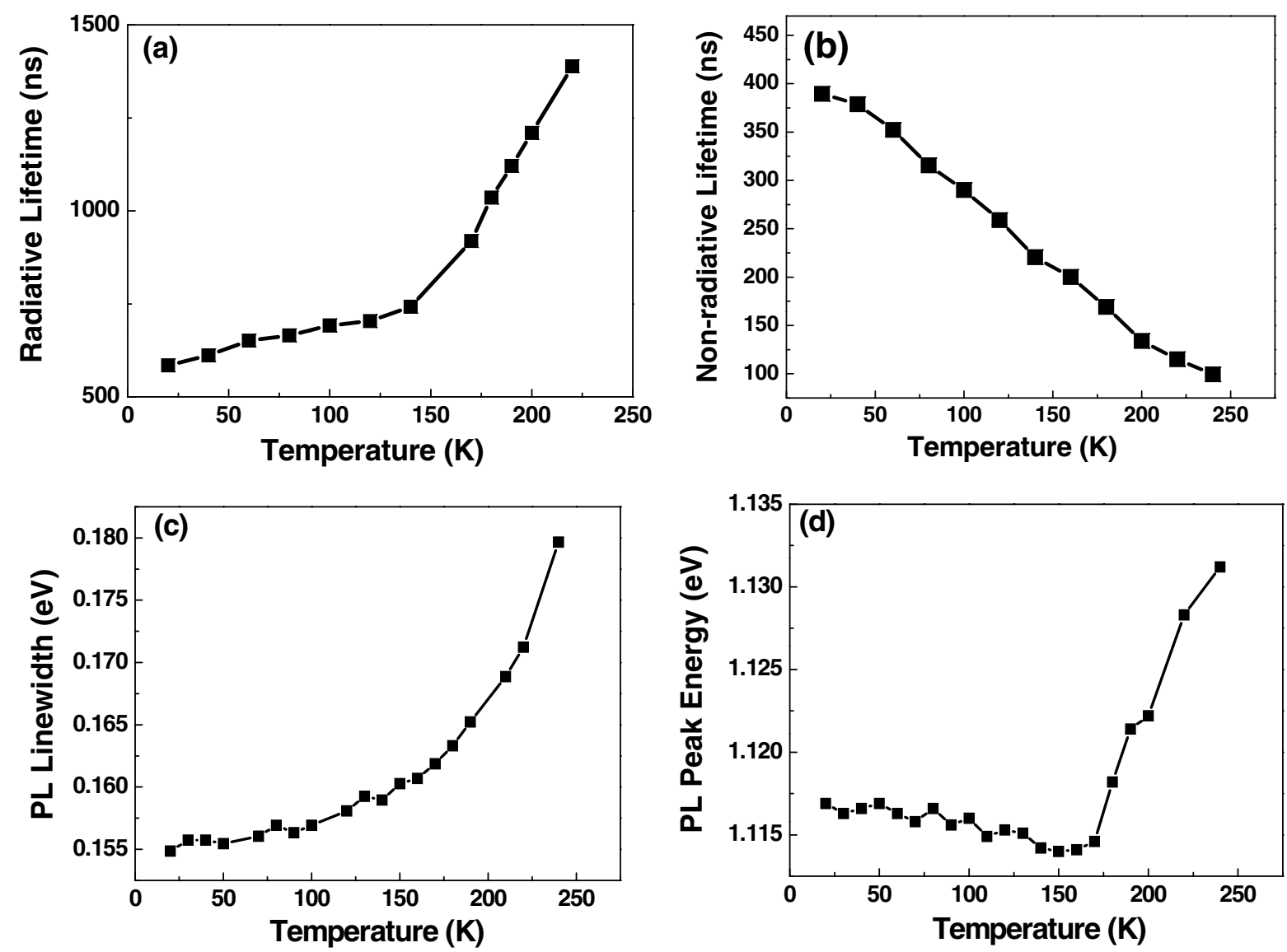

Figure 2. (a) Dependence of the radiative lifetime on temperature. (b) Dependence of the non-radiative lifetime on temperature. (c) Dependence of the spectra linewidth and (d) peak energy of photoluminescence spectra on temperature.

and (b). It is found that the radiative lifetime increases from 600 to 1400 ns while the non-radiative lifetime decreases from 400 to $100 \mathrm{~ns}$ with increasing temperature from 20 to 240 $\mathrm{K}$. The decrease of the non-radiative lifetime with increasing temperature arises from the exciton dissociation as will be discussed later.

We now attempt to understand the underlying mechanisms responsible for the recombination dynamics in $\mathrm{CdTe} / \mathrm{CdSe}$ QDs. In our experiment, the sample has been excited with a much lower laser power of about $90 \mu \mathrm{W}$ with a spot diameter of about $4 \mathrm{~mm} \mathrm{[18].} \mathrm{Therefore,} \mathrm{the} \mathrm{average} \mathrm{number} \mathrm{of} \mathrm{excited}$ excitons in each dot is very small (single-exciton regime) due to the very small photon flux density [19]. Under the singleexciton regime, we can neglect the Auger scattering effect and exciton-exciton interaction, which may contribute to the multiexciton effects in type-II colloidal QDs $[18,20]$. In order to have a deeper understanding of the recombination behavior, we have performed the temperature-dependent analysis. As shown in figure 2(a), the temperature dependence of the radiative lifetime contains two different regions. According to previous reports, it is known that the radiative dynamics is dominated by delocalized excitons at low temperature and is caused by the mixture of delocalized excitons, electrons and holes at high temperature [11, 21-23]. The increased radiative lifetime with increasing temperature is attributed to the reduced oscillator strength of the PL transition caused by the increased carrier-phonon interaction [21, 22]. The rapidly increased radiative lifetime in the high temperature region can be attributed to the enhanced carrier-phonon interaction and the dissociation process of the delocalized excitons into electrons and holes [11, 23]. In order to confirm our assumption, let us look at the temperature dependence of steady-state PL spectra. As shown in figures 2(c) and (d), the linewidth and the peak energy also exhibit two different regions of temperature dependence. These results indicate that the temperature dependence of steady-state PL spectra are consistent with those of the temperature dependence of the radiative lifetime. It is worth noting that there exists a slow increase of linewidth as well as a slight decrease of PL peak energy with increasing temperature. These behaviors possibly arise from carrier-phonon interaction and thermal relaxation of delocalized excitons into energetically low-lying states [11].

As described above, delocalized excitons are gradually dissociated with increasing temperature in the high temperature region. After dissociation, the resultant electrons and holes are able to move to non-radiative states. In turn, it will affect the behaviors of the temperature-dependent integrated PL intensity and non-radiative lifetime. As shown in figure 3 , it is clear that the temperature-dependent non-radiative lifetime can be well fitted by the Arrhenius equation:

$$
\frac{1}{\tau_{\text {nrad }}}=\frac{\exp \left(-E_{\mathrm{A}} / k T\right)}{\tau_{t}}
$$




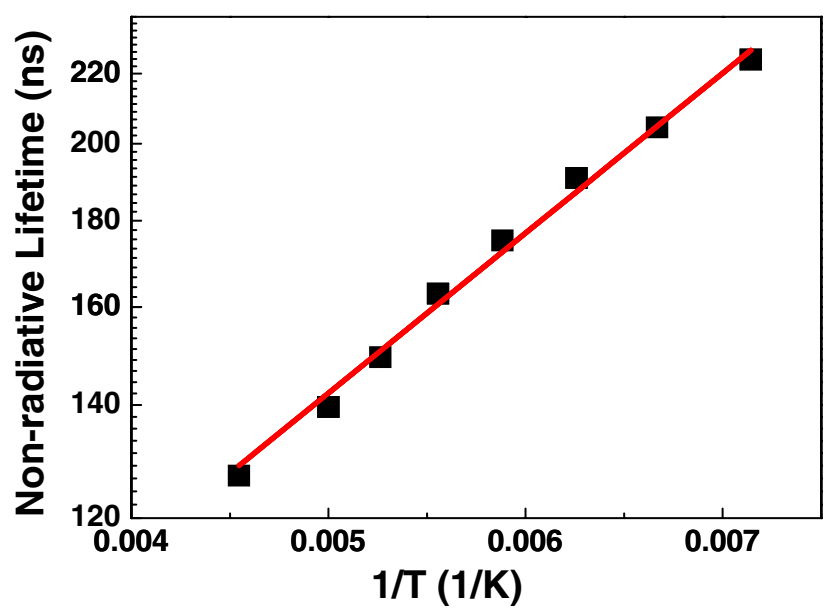

Figure 3. Arrhenius plot of the non-radiative lifetime and the activation energy fit.

where $1 / \tau_{t}$ is the rate of the dissociation and the traveling of carriers to a non-radiative state and $E_{\mathrm{A}}$ is the corresponding thermal activation energy. With the fitting parameter $2.1 \times$ $10^{7} \mathrm{~s}^{-1}$ of $1 / \tau_{t}$, the obtained activation energy of $19.2 \mathrm{meV}$ is in good agreement with the exciton binding energy in type-II CdTe/CdSe QDs studied earlier [11].

Figure 4(a) shows the variation of the radiative lifetime with different detected PL energies at $20 \mathrm{~K}$. It is found that the radiative lifetime increases gradually with decreasing detected photon energy within all the regions of photon energy. This result is quite different from other reports for the recombination dynamics of localized carriers, in which the increase of radiative lifetime eventually saturates with decreasing detected energy in the low photon energy region [24, 25]. However, it is consistent with the above observation that the recombination dynamics is dominated by the relaxation of delocalized excitons in the low temperature region. To understand the recombination mechanism further, following the previous reports [26, 27], we attribute the large PL linewidth to the size disorder in our sample. According to the previous study $[10,11]$, we can estimate the corresponding size of the CdTe/CdSe QDs with different emission photon energy. Figure 4(b) depicts the radiative lifetime as a function of the size of QDs with the size distribution from 6.8 to $9.2 \mathrm{~nm}$, which is caused by the uncertainty in the colloidal synthesis process [10]. It shows that the radiative lifetime strongly decreases on reducing the size of QDs, and follows a powerlaw relationship with a power factor of 3.30. This behavior can be understood in accordance with the theoretical prediction of the quantum confinement effect [28]. Based on quantum confinement, the oscillator strength and exciton energy in QDs are separately proportional to the size $[29,30]$ and square of the size, respectively [31]. Theoretically, the radiative lifetime is inversely proportional to the product of the energy squared and the oscillator strength [32]. Therefore, the radiative lifetime is proportional to the cube of the size, which is in good agreement with the relationship between the radiative lifetime and the size of QDs as shown in figure 4(b).

Quite interestingly, we also observe that the radiative decay rate increases rapidly under a moderate excitation power
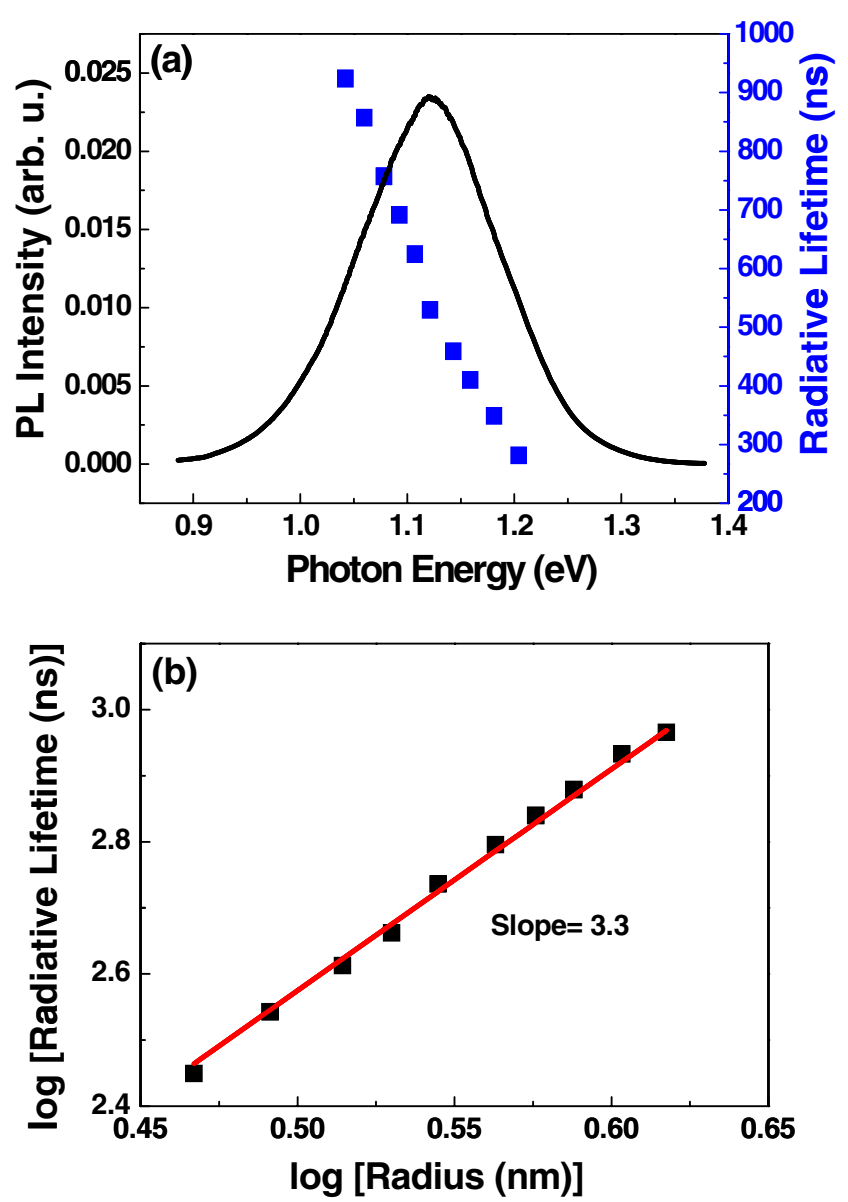

Figure 4. (a) Dependence of the radiative lifetime on detected photoluminescence energy. Solid line shows the photoluminescence spectrum at $20 \mathrm{~K}$. (b) Logarithmic plot of the radiative lifetime as a function of the size of $\mathrm{CdTe} / \mathrm{CdSe}$ quantum dots.

at $20 \mathrm{~K}$ as shown in figure 5(a). For example, the radiative decay rate increases from $3 \times 10^{6}$ to $1.4 \times 10^{7} \mathrm{~s}^{-1}$ when the pumping intensity changes from 1 to $10 \mathrm{~mW} \mathrm{~cm}^{-2}$. This strong variation of the radiative decay rate will eventually stop when the excitation power increases further, as shown in figure 5(a). This behavior can be rationalized by the type-II band alignment associated with CdTe/CdSe QDs [11], which causes the spatial separation of electrons and holes and creates the band bending effect. For clarity of elucidation, the underlying mechanism based on a band bending model is illustrated in figure 5(b). In contrast to type-I semiconductor structures, in a type-II band structure, the energy minima for electrons and holes lie in different materials. Due to the typeII band alignment, the photo-excited electrons and holes are confined at the core-shell interface. The separation of electrons and holes builds up an internal electric field and the conduction band and valence band start to bend and subsequently enhance the overlap of the wavefunction of electrons and holes $[1,2]$. Since the PL is generated from the recombination of the accumulated electrons and holes at the interface, an increase in the excitation power increases the wavefunction overlap of electrons and holes, and results in an enhanced radiative decay rate $[33,34]$. 


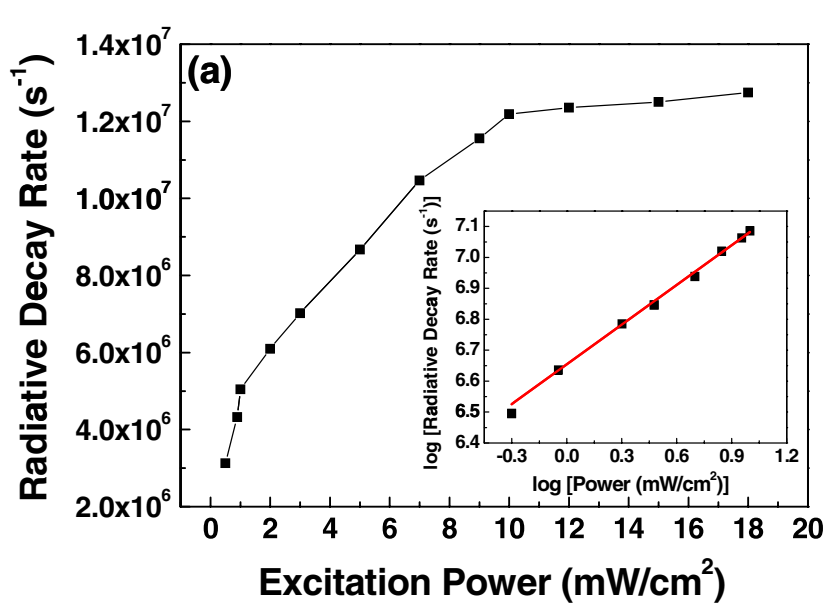

(b)

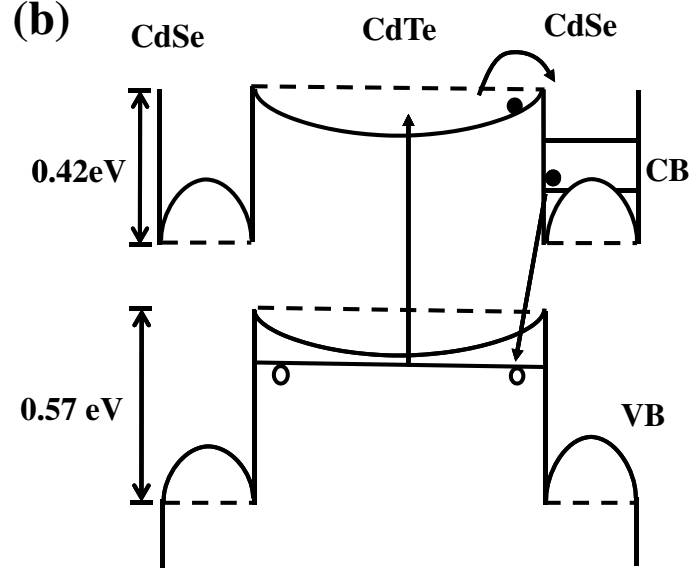

Figure 5. (a) Dependence of the radiative decay rate on the excitation power. The inset shows the dependence of the radiative decay rate on the squared root of the excitation power. (b) A type-II band structure under the influence of the band bending effect. The band bending can trap electrons and holes near the interface, and the quantum confinement will cause the increase in the radiative decay rate.

In order to quantify our observation, let us consider the wavefunction overlap integral of electrons and holes, given by the following expression [35]:

$$
\begin{aligned}
& I\left(\varepsilon, \varepsilon^{\prime}\right)=4 \pi \int_{a}^{\infty} \psi_{\mathrm{e}}(r) \psi_{\mathrm{h}}(r) r^{2} \mathrm{~d} r \\
& =\sqrt{\frac{2 m_{\mathrm{h}} \varepsilon e F(a)}{a\left(\Delta E_{\mathrm{C}}-\varepsilon\right)}} \frac{\hbar}{m_{\mathrm{h}} \varepsilon^{\prime}+m_{\mathrm{e}}\left(\Delta E_{\mathrm{C}}-\varepsilon\right)},
\end{aligned}
$$

where $F(a)$ is the internal electric field, $a$ is the radius of CdTe, $m_{\mathrm{e}}\left(m_{\mathrm{h}}\right)$ is the effective mass of the electron (hole), $\varepsilon\left(\varepsilon^{\prime}\right)$ is the dielectric constant of $\mathrm{CdSe}(\mathrm{CdTe})$ and $\Delta E_{\mathrm{C}}$ is the conduction band offset. The relationship between carrier densities of electrons $n_{w}$ and holes $p_{w}$ and excitation intensity can be described by equation [33]:

$$
n_{w} p_{w}=n_{w}^{2}=\frac{\alpha(L+l)}{\gamma} I,
$$

where $\alpha$ denotes the absorption coefficient, $L$ is the thickness of the CdSe layer, $l$ is the diameter of CdTe, $\gamma$ is the radiative recombination coefficient and $I$ is the excitation intensity. The strongly localized carriers near the interface will produce an approximately triangular well with the electric-field strength of [33]

$$
F(a)=\frac{2 \pi e n_{w}}{\varepsilon_{0}} \propto I^{1 / 2} .
$$

Because the radiative decay rate is proportional to the square of the wavefunction overlap, it is therefore linearly proportional to the square root of the excitation intensity. The inset of figure 5(a) depicts the logarithmic plot of the radiative decay rate as a function of the excitation intensity and the slope of the fitting curve is 0.44 , which is close to the theoretical value of 0.5. Therefore, the inherent nature of a type-II band alignment can really alter the recombination dynamics of photo-excited carriers.

\section{Conclusions}

In conclusion, we have investigated the recombination dynamics in CdTe/CdSe type-II QDs. The measured PL decay time of about several hundred nanoseconds shows the slow recombination nature due to the weak wavefunction overlap of the spatially separated electrons and holes in a type-II heterostructure. The linear dependence of the radiative lifetime on temperature below $150 \mathrm{~K}$ demonstrates the dominance of delocalized exciton recombination and the non-radiative process is mainly dictated by the dissociation of delocalized excitons. By measuring the radiative lifetimes at different detected photon energies, we demonstrate that the radiative lifetime is approximately proportional to the cube of the size of QDs, which can be well accounted for by the quantum confinement effect. Finally, the dependence of the recombination dynamics on excitation power reveals the band bending effect arising from the inherent character of a type-II band alignment.

\section{Acknowledgments}

This work was supported by the Ministry of Education and National Science Council of the Republic of China.

\section{References}

[1] Larsson M, Elfving A, Holtz P O, Hansson G V and Ni W X 2003 Appl. Phys. Lett. 824785

[2] Chiu Y S, Ya M H, Su W S and Chen Y F 2002 J. Appl. Phys. 925810

[3] Hatami F et al 1998 Phys. Rev. B 574635

[4] Zaitsev S V, Maksimov A A, Kulakovskii V D, Tartakovskii I I, Yakovlev D R, Ossau W, Hansen L and Landwehr G 2002 J. Appl. Phys. 91652

[5] Bimberg D and Ledentsov N 2003 J. Phys.: Condens. Matter 15 R 1063

[6] Pettersson H, Btááh L, Carlsson N, Seifert W and Samuelson L 2001 Appl. Phys. Lett. 7978

[7] Gur I, Fromer N A, Geier M L and Alivisatos A P 2005 Science 310462

[8] Kim S et al 2004 Nat. Biotechnol. 2293

[9] Kim S, Fisher B, Eisier H J and Bawendi M 2003 J. Am. Chem. Soc. 12511466 
[10] Chou P T, Chen C Y, Cheng C T, Pu S C, Wu K C, Cheng Y M, Chou Y H and Chiu H T 2006 ChemPhysChem 7222

[11] Wang C H, Chen T T, Tan K W, Chen Y F, Cheng C T and Chou P T 2006 J. Appl. Phys. 99123521

[12] Lee W Z, Shu G W, Wang J C, Shen J L, Lin C A, Chang W H, Ruaan R C, Chou W C, Lu C H and Lee Y C 2005 Nanotechnology 161517

[13] Schlegel G, Bohnenberger J, Potapova I and Mews A 2002 Phys. Rev. Lett. 88137401

[14] Koole R, Liljeroth P, de Mello Donegá C, Vanmaekelbergh D and Meijerink A $2006 \mathrm{~J}$. Am. Chem. Soc. 12810436

[15] Crooker S A, Hollingsworth J A, Tretiak S and Klimov V I 2002 Phys. Rev. Lett. 89186802

[16] Fuster D, Martínez-Pastor J, González L and González Y 2005 Phys. Rev. B 71205329

[17] Gurioli M, Vinattieri A, Colocci M, Deparis C, Massies J, Neu G, Bosacchi A and Franchi S 1991 Phys. Rev. B 443115

[18] Oron D, Kazes M and Banin U 2007 Phys. Rev. B 75035330

[19] Valerini D, Cretí A, Lomascolo M, Manna L, Cingolani L and Anni M 2005 Phys. Rev. B 71235409

[20] Piryatinski A, Ivanov S A, Tretiak S and Klimov V I 2007 Nano Lett. 7108

[21] Lefebvre P, Allègre J, Gil B, Kavokine A, Mathieu H, Kim W, Salvador A, Botchkarev A and Morkoc H 1998 Phys. Rev. B 57 R9447
[22] Narukawa Y, Kawakami Y, Fujita S and Nakamura S 1999 Phys. Rev. B 5910283

[23] Im J S, Moritz A, Steuber F, Härle V, Scholz F and Hangleiter A 1997 Appl. Phys. Lett. 70631

[24] Satake A, Masumoto Y, Miyajima T, Asatsuma T, Nakamura F and Ikeda M 1998 Phys. Rev. B 57 R2041

[25] Strassburg M, Dworzak M, Born H, Heitz R, Hoffmann A, Bartels M, Lischka K, Schikora D and Christen J 2002 Appl. Phys. Lett. 80473

[26] Peng X, Wickham J and Alivisatos A P 1998 J. Am. Chem. Soc. 1205343

[27] Swaminathan P, Antonov V N, Soares J A N T, Palmer J S and Weaver J H 2006 Phys. Rev. B 73125430

[28] Javier A, Magana D, Jennings T and Strouse G F 2003 Appl. Phys. Lett. 831423

[29] Leatherdale C A, Woo W K, Mikulec F V and Bawendi M G 2002 J. Phys. Chem. B 1067619

[30] Zorman B and Friesner R A 2003 J. Chem. Phys. 1185937

[31] Borrelli N F, Hall D W, Holland H J and Smith D W 1987 J. Appl. Phys. 615399

[32] Turro N J 1978 Modern Molecular Photochemistry (Menlo Park, CA: Benjamin/Cummings)

[33] Ledentsov N N et al 1995 Phys. Rev. B 5214058

[34] Sun C K, Wang G, Bowers J E, Brar B, Blank H R, Kroemer H and Pilkuhn M H 1996 Appl. Phys. Lett. 681543

[35] Shik A, Ruda H and Sargent E H 2001 Nanotechnology 12523 